\title{
Molecular overlap links tuberous sclerosis, fragile $X$
}

\author{
BY RACHEL ZAMZOW
}

16 AUGUST 2021

\section{Listen to this story:}

https://www.spectrumnews.org/wpcontent/uploads/2021/08/audio-68daabcb-5e35-4600-9c90-b77ddl3f149a9-encodings.mp3

Brain cells from the cerebellums of mice that model tuberous sclerosis complex (TSC) show dampened protein levels, according to a new study. The affected proteins' levels are controlled by FMRP, the protein missing in people with fragile $\mathrm{X}$ syndrome - a result that suggests the two conditions share molecular roots.

"We have been sort of thinking even prior to this study that there might be some overlap at the cellular level between FMRP and TSC," says lead researcher Mustafa Sahin, professor of neurology at Harvard University. He and his team previously showed that cerebellar cells derived from people with TSC have reduced levels of FMRP and its targets.

"This was another way of showing that it could also happen in the cerebellum," Sahin says of the new work.

Like fragile $X$ syndrome, TSC is characterized by intellectual disability and often autism. The condition arises from mutations in either the TSC1 or TSC2 genes, which yield proteins needed to keep a check on the mTOR signaling pathway. Without regulation, this pathway operates in overdrive, boosting protein production and cell proliferation.

The new study suggests that the mTOR pathway itself regulates FMRP, which is known to bind to RNA transcripts and stall their translation into proteins. But the mechanism connecting mTOR and FMRP remains unknown, says Angelique Bordey, professor of neurosurgery and of cellular and molecular physiology at Yale University, who was not involved in the study. 


\section{Spectrum | Autism Research News}

https://www.spectrumnews.org

"It's a set of data and a study that actually open new doors and require additional investigations," she says.

\section{Genetic decline:}

Sahin and his colleagues engineered mice that lack the TSC1 gene in a class of cerebellar cells called Purkinje cells and measured RNA levels in these cells to gauge gene expression.

Mice lacking TSC1 showed diminished gene expression compared with controls, the researchers found. In fact, among the genes that differed between the groups, 72 percent were downregulated in the model mice. The findings appeared 14 July in eLife.

The RNA fragments in the TSC1 mice were also more vulnerable to decay than those of controls, which may explain the animals' downgraded gene expression. And the excess RNA degradation applied particularly to a core group of genes that are regulated by FMRP and help support synapses, the team found. Many are also tied to autism.

Despite having lower levels of RNA overall, the Purkinje cells lacking TSC1 showed no change in the amount of RNA attached to ribosomes - the cellular machines that convert RNA into proteins.

The findings hint that the cells without TSC1 are more efficient at loading RNA onto ribosomes, which is puzzling, Sahin says. "It almost seems like the cell is trying to balance itself back out in terms of trying to increase the protein expression of these transcripts."

But this purported compensatory effort falls short: The protein levels of three FMRP target genes are reduced in the cerebellar cells missing TSC1, the researchers found. One protein in particular, SHANK2, resides in the signal-receiving end of synapses, and mutations in the gene encoding SHANK2 are commonly linked to autism and intellectual disability.

Mice lacking TSC1 in their Purkinje cells display autism-like traits, such as reduced social encounters, repetitive behaviors and motor dysfunction - all of which disappear after treatment with the drug rapamycin, which inhibits mTOR, a 2012 study by Sahin's lab showed.

The new study points to possible molecular underpinnings of these features.

"It suggests that some of the transcripts that are dysregulated could potentially be involved in some of the neurological deficits," Bordey says, but it will be key to test whether reinstating these RNA levels rescues the animals' behaviors.

\section{Protein patterns:}

Taken together, the findings add to mounting evidence that TSC mutations lead to lower protein 


\section{Spectrum | Autism Research News}

https://www.spectrumnews.org

levels in some cases.

"It goes against the concrete expectation that there's a global increase in translation, but I think maybe sometimes people have a premature conclusion on that," Bordey says.

For example, living mice engineered to lack TSC2 show diminished protein production throughout the brain, according to a 2018 study. But the effects of TSC mutations vary by cell type, says Carolyn Beebe Smith, senior investigator at the U.S. National Institute of Mental Health, who led that work.

Another team uncovered a different pattern in the hippocampus two years later: Mice lacking TSC2 there have increased expression of FMRP-linked genes in this brain region.

"I think we also have to consider that isolated neurons may not be representative of what's going on in an intact nervous system," Beebe Smith says.

Regardless, the new study strengthens the ties between TSC and fragile X syndrome. The findings hint that the two conditions share not only behavioral features, but also molecular paths.

Exploring this convergence in people could help researchers uncover the conditions' molecular origins, as well as how their different treatment options may overlap, Sahin says. For example, upcoming clinical trials plan to test if drugs that help some people with fragile $X$ syndrome, such as mGluR5 antagonists, also benefit those with TSC.

"Until we actually go to the patients and test some of these hypotheses, unfortunately, drawing conclusions from slice culture or these kind of transcriptome experiments [is] going to be very difficult," he says. "At the end of the day, it's the patients that matter."

Cite this article: https://doi.org/10.53053/CCSY9613 